\title{
量子密钥传输系统的主动相位补偿
}

\author{
陈 巍 ${ }^{\circledR 0}$ 韩正甫 $^{\Phi^{*}}$ 莫小范 $^{\oplus}$ 许方星 ${ }^{\circledR}$ 卫 国 ${ }^{\circledR}$ 郭光灿 $^{\oplus}$ \\ (1) 中国科学技术大学中国科学院量子信息重点实验室, 合肥 230026; (2) 中国科学技术大学电子工程与信息科学系, 合肥 230027 . \\ *联系人, E-mail: zfhan@ustc.edu.cn)
}

\begin{abstract}
摘要 量子密钥分配系统的稳定性是影响其实用性能的重要因素之一. 除光纤自身固有的双折射效应 外, 环境造成的相位漂移也会严重影响密钥传输系统的性能. Faraday-Michelson (F-M)量子密钥分配系 统可以自适应地补偿光纤双折射造成的影响, 但是在实际应用中, 相位漂移仍然会对系统性能产生较 大影响. 本文详细分析了在实际中造成F-M系统相位漂移的主要原因, 并提出了一种高效的主动相位搜 索和补偿方案. 应用这一方案, Faraday-Michelson系统在脱离光学平台的实际条件下进行了长时间稳定 的量子密钥分配. 实验的传输距离大于 $37 \mathrm{~km}$, 系统平均误码率 $1.59 \%$, 误码波动标准差 $0.46 \%$. 结果表 明这一主动相位补偿技术在不增加额外器件的条件下，可用于以双不等臂Mach-Zehnder(AMZ)干涉仪 为基础的实际量子密钥分配系统.
\end{abstract}

关键词 量子密钥分配 Faraday-Michelson 系统 相位漂移 主动相位补偿

量子密钥分配(Quantum Key Distribution)系统是 新一代密钥分配方案, 它的安全性由物理原理而非 算法强度所保证. 自Bennet和Brassard在 1984 年提出 BB84 方案后 $[1]$, 量子密码系统在理论和实验研究上 都取得了长足的进展 ${ }^{[2 \sim 7]}$.

近年来随着网络安全的要求不断提高, 许多光 纤量子密码系统应运而生. 相比偏振编码来说, 光子 信号在光纤中传输时其相位信息更易保持, 因此绝 大多数现有的光纤量子密码系统都采用相位编码方 案实现 ${ }^{[8]}$. 光纤量子密码系统要在实际条件下长时 间稳定工作，必须要能够应对光纤的双折射效应以 及环境变化所造成的相位漂移. 莫小范等人于 2005 年提出的“法拉第-迈克耳逊”(Faraday-Michelson, F-M)系统 [9]可以自适应地补偿AMZ干涉环的双折射 效应, 但是在实际的QKD通信过程中仍然存在相位 漂移.

目前针对 AMZ 型干涉系统的相位漂移主要有以 下 3 种解决方案.

（i ）改进干涉环的结构来以适应的补偿相位漂 移, 如“plug-and-play”结构 ${ }^{[10]}$.

（ii）被动补偿: 采用良好的隔温和避震措施降 低环境对干涉系统的影响.

(iii) 主动补偿: 采用主动搜索获取相位漂移的
参数并加以补偿.

“Plug-and-play”系统对于光纤的双折射效应和相 位漂移都有很好的自补偿作用. 但是该方案容易受 到特洛伊木马攻击 ${ }^{[4,11,12]}$, 其往返式的设计使得该系 统的原始传输码率低于相同条件下的单向系统. 被 动补偿包括采用低的热膨胀系数材料来制作干涉环 [13]、采用集成工艺刻蚀干涉环、增加减震装置并且进 行精确的温度控制 [14,15]. 这种方案可以降低温度漂 移及其带来的影响, 但无法从原理上完全消除温度 漂移. 在此基础上, Nambu等人 ${ }^{[16]}$ 提出了一种改进的 被动控制方案, 通过精确控制AMZ干涉环的温度, 使干涉环的长短臂相位差保持为 $2 \pi$ 的整数倍. 虽然 这些方法可以在一定程度上降低环境的干扰，但无 法从本质上消除相位漂移，此外还需要增加控制精 度在 $0.01^{\circ} \mathrm{C}$ 以内的温控系统. 即便采用了这些措施, 由于发射和接收端的干涉环温度的相对独立变化, 仍然可以在实验中观察到相位漂移现象. Towsend ${ }^{[17]}$ 和Yuan等人 ${ }^{[18]}$ 采用调节光纤臂长的方式对相位进行 主动补偿, Yuan等人 ${ }^{[18]}$ 和Zavaryev等人 ${ }^{[19]}$ 采用单独 的强参考光来测量系统相位漂移参数; Makarov等人 ${ }^{[20]}$ 提出了单光子计数方法的软件补偿方案, 但未进 行实际的量子密码实验. 调节光纤臂长的方法速率 较慢, 限制了其在高速 $\mathrm{QKD}$ 系统中的应用, 同时额 
外器件将增加干涉环的复杂度和成本; 使用单独的 强参考光会增加光学系统的复杂度, 同时容易引入 不必要的干扰; 单光子计数的方案随传输距离的增 加, 获得足够计数的时间相应增加, 因此可补偿范围 随距离增加而减小.

QKD 系统在脱离光学平台的实际环境中运行时, 环境所造成的相位漂移将更加明显. 为了使 QKD 系 统以较低误码率长时间稳定运行, 需要采用高效的 算法获取相位漂移参数并进行补偿. 本文详细分析 了 F-M 系统在实际条件下相位漂移的产生原因, 并 提出了一种快速的相位补偿方案. 应用这一方法实 现了 F-M 系统在实际应用环境中长期(测试时间 $18 \mathrm{~h}$ ) 无人值守的稳定传输, $37 \mathrm{~km}$ 传输距离下系统长期平 均误码率为 $1.59 \%$, 误码波动标准差小于 $0.46 \%$. 该 方法不仅可用于 F-M 系统, 也可以推广用于一切以 不等臂 Mach-Zehnder 干涉系统为基础的量子密码系 统.

\section{Faraday-Michelson 系统的相位漂移}

F-M 系统可以等效为图 1 干涉系统.

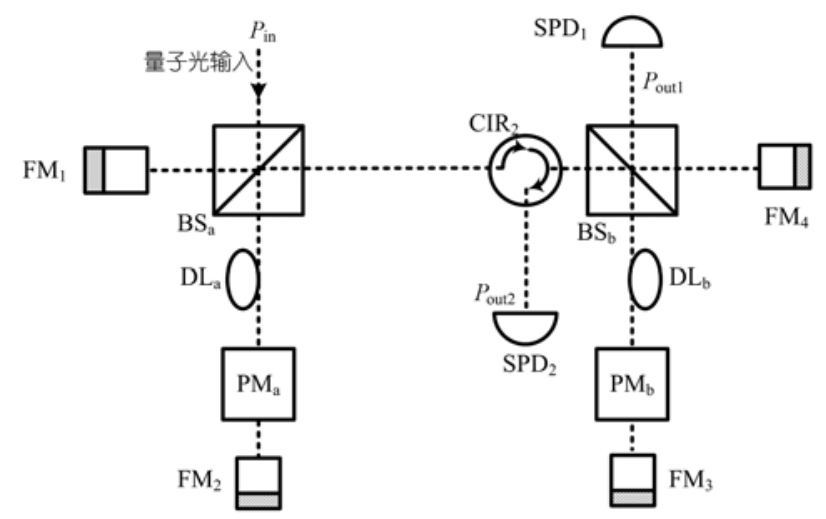

图 1 F-M 系统等效光路

$B S$ 为 $50 / 50$ 光纤分束器; FM 为 Faraday 反射镜; SPD 为单光子探测器; CIR 为环行器; DL 为光纤延时线

对其光路进行分析可以得到 $P_{\text {out } 1}$ 的输出表达式 为

$$
P_{\text {out } 1}=\frac{\alpha}{8}\left[1+\cos \left(\Delta \varphi_{p l}+\Delta \varphi_{p m}\right)\right] E_{\text {in }}^{+} E_{\text {in }},
$$

式中 $\alpha$ 为光学系统损耗; 发生干涉的两个光脉冲在发 射端和接收端干涉环内所走的光程差 $\Delta \varphi_{p l}=k\left(\Delta l_{\mathrm{b}}\right.$ $\left.-\Delta l_{\mathrm{a}}\right), \Delta l_{\mathrm{a}}$ 和 $\Delta l_{\mathrm{b}}$ 分别为 Alice 和 Bob 的干涉环长短臂 之差, 波矢 $k=\frac{2 \pi}{\lambda} ; \Delta \varphi_{p m}$ 代表发射端和接收端调相 器调制的相位差. 当 $\Delta \varphi_{p l}+\Delta \varphi_{p m}=0$ 时, $P_{\text {out } 1}$ 输出达到
最大, $\Delta \varphi_{p l}+\Delta \varphi_{p m}=\pi$ 时最小.

在不进行相位补偿时, 由(1)式导出相位误差造 成的误码率为:

$$
Q B E R_{\Delta \varphi}=\sin ^{2}\left(\frac{\Delta \varphi_{e}}{2}\right),
$$

其中 $\Delta \varphi_{e}=\Delta \varphi_{e p l}+\Delta \varphi_{e p m}, \Delta \varphi_{e p l}$ 是发射端和接收端干涉 环不对称所造成的相位误差, $\Delta \varphi_{\text {epm }}$ 为发射端和接收 端调相器的基准相位差. 如果 $\Delta \varphi_{e p l}$ 保持稳定且满足 相干条件, 则可以精确调节 $\Delta \varphi_{e p m}$ 使 $\Delta \varphi_{e}$ 最小, 从而探 测器 $\mathrm{SPD}_{1}$ 的计数最大且保持稳定. 但是在实际测试 中发现, 即使调节 Alice 和 Bob 的调相电压使 $\Delta \varphi_{e} \rightarrow 0$, 从而 $P_{\text {out } 1}$ 计数达到最大, 在系统运行的过程中探测 器的计数仍然会发生明显超出涨落范围之外的波动, 即F-M系统在实际运行中存在相位漂移. 相位漂移的 根本原因是环境的变化, 如温度的波动, 机械震动等 导致干涉环的光程产生变化, 从而 $\Delta \varphi_{p l}$ 发生改变. 通 过实际运行的观测, 温度变化对F-M系统的影响远远 大于机械震动, 是降低系统可见度并导致误码的主 要因素, 因此下面主要分析温度变化造成的相位漂 移.

设光纤的热膨胀系数为 $\alpha_{T}$, 温度变化量为 $\Delta_{T}$, 光 纤长度为 $l$, 则温度变化造成的光纤长度改变 $\Delta L=$ $\alpha_{T} \cdot l \cdot \Delta_{T}$. 如果将制作干涉环时的环境温度定为参考 温度 $T_{0}$, Alice 和 Bob 工作时的环境温度相对于 $T_{0}$ 的 变化分别为 $\Delta T_{\mathrm{a}}=T_{\mathrm{a}}-T_{0}$ 和 $\Delta T_{\mathrm{b}}=T_{\mathrm{b}}-T_{0}$, 则在考虑温度 变化的情况下, 发生干涉的两个脉冲相位差可以表 示为

$$
\Delta \varphi=\Delta \varphi_{\mathrm{pm}}+k\left(\Delta l_{\mathrm{b}}-\Delta l_{\mathrm{a}}\right)+\alpha_{\mathrm{T}} k\left(\Delta \mathrm{l}_{\mathrm{b}} \Delta T_{\mathrm{b}} \Delta l_{\mathrm{a}} \Delta T_{\mathrm{a}}\right),
$$
式中的 $\Delta l_{\mathrm{a}}$ 和 $\Delta l_{\mathrm{b}}$ 为干涉环在设计时的额定臂长差, 式 中第三项为环境温度变化对相位的影响. 定义 $l=$ $\left(\Delta l_{\mathrm{a}}+\Delta l_{\mathrm{b}}\right) / 2, T=\left(\Delta T_{\mathrm{a}}+\Delta T_{\mathrm{b}}\right) / 2, \Delta l=\Delta l_{\mathrm{b}}-\Delta T_{\mathrm{a}}$ 代表 Alice 和 Bob 干涉环固有的不对称性, $\Delta T=\Delta l_{\mathrm{b}}-\Delta T_{\mathrm{a}}$ 代表 Alice 和 Bob 环境温度的差异. 则(3)式可以改写为

$$
\begin{aligned}
\Delta \varphi & =\Delta \varphi_{p m}+k \Delta l+k \alpha_{T} T \Delta l+k \alpha_{T} l \Delta T \\
& =\Delta \varphi_{p m}+\Delta \varphi_{0}+\Delta \varphi_{T 1}+\Delta \varphi_{T 2} \\
& =\Delta \varphi_{p m}+\Delta \varphi_{0}+\Delta \varphi_{\mathrm{var}} .
\end{aligned}
$$

式中 $\Delta \varphi_{p m}$ 对应调相器的相位差, $\Delta \varphi_{0}$ 是干涉环固有的 不对称性所带来的常量相位差, $\Delta \varphi_{T 1}$ 和 $\Delta \varphi_{T 2}$ 是环境变 化相关项, 他们可以合并为相位漂移 $\Delta \varphi_{\mathrm{var}}$. 以我们设 计的干涉环为例: 干涉环的等效长短臂之差约为 $3 \mathrm{~m}$, 石英光纤的热膨胀系数为 $5.4 \times 10^{-7}{ }^{\circ} \mathrm{C}$, 按照(4)式和 (2)式计算, $20.03{ }^{\circ} \mathrm{C}$ 的温度变化就将引起 $1 \%$ 的系统误 
码, 为了能使系统稳定运行, 必须对相位漂移进行有 效补偿.

\section{2 主动相位补偿}

相位编码 QKD 系统大多遵循 BB84 或者 B92 等 QKD 协议, 采用主动相位调制. 调相器需要确定 $\Delta_{p m}$ 分别等于 $0, \pi / 2, \pi$ 和 $3 \pi / 2$ 时的调制工作点, 对于电控 调相器来说就是它的电压工作点. 如果可以通过主 动搜索获得相位漂移的参数, 即可通过调整工作点 来补偿相位漂移.

主动相位补偿分为相位漂移参数获取和补偿两 个过程. 每次密钥分配操作前, 系统首先进行“扫描” 操作以获取相位漂移参数并确定补偿参数 [21]. 扫描 的流程如下: 发射端Alice将发射端调相器 $\mathrm{PM}_{\mathrm{a}}$ 的调 相电压固定为 $V_{\mathrm{a} 0}$, 接收端Bob以此时 $\mathrm{PM}_{\mathrm{a}}$ 调制的相位 $\varphi_{a 0}$ 作为参考相位. 由于在 $\mathrm{QKD}$ 中, Alice和Bob相位差 才能代表信息, 因此不妨假定 $V_{\mathrm{a} 0}=0, \varphi_{\mathrm{a} 0}=0$. Bob控 制接收端调相器 $\mathrm{PM}_{\mathrm{b}}$ 的调相电压从 $V_{\min }$ 以步长 $\Delta V$ 扫描 到 $V_{\text {max }}, V_{\text {min }}$ 到 $V_{\text {max }}$ 至少需覆盖 $2 \pi$ 的相位范围. 在每一 个电压值 $V_{i}$ 上系统等待 $N$ 个同步脉冲, 并对单光子探 测器的计数进行累加, 得到计数 $C_{i}$. 由此可以得到一 组数据 $\left\{V_{i}, C_{i}\right\}$, 这组“电压-单光子计数”曲线即为“干 涉条纹”, 如图 2. 从(1)式和图 2 可以看出, 干涉条纹 符合正弦曲线模型. 在正弦曲线的最高点, $\mathrm{PM}_{\mathrm{a}}$ 和 $\mathrm{PM}_{\mathrm{b}}$ 的相位相等; 在曲线的最低点两者相位相差 $\pi$. 计数最高点对应的电压 $V_{C_{-} \max }$ 和最低点对应的电压 $V_{c \_ \text {min }}$ 之差即为调相器的半波电压 $V_{\text {half }}$.

为了计算 Bob 的工作电压, 首先需要确定一个 参考电压作为基准. 如果选择与 $V_{\mathrm{a} 0}$ 所调制的相位相

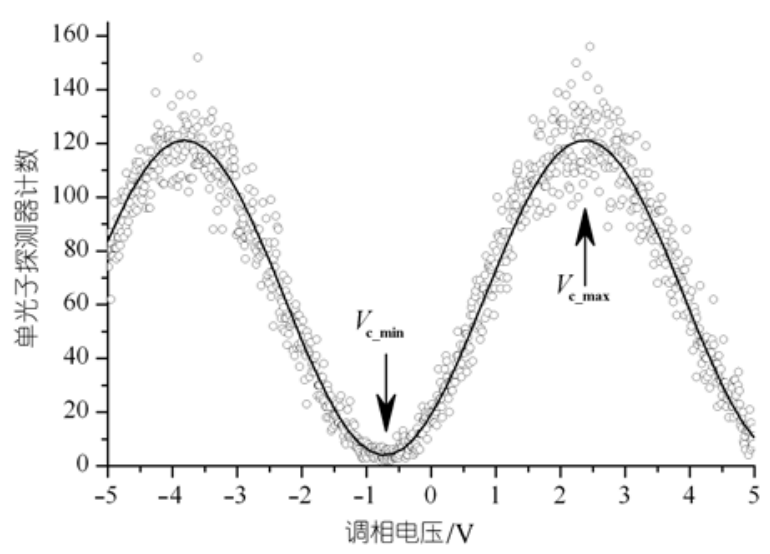

图 2 扫描得到的干涉条纹

扫描电压从 $-5 \mathrm{~V}$ 到 $+5 \mathrm{~V}$, 电压步长 $10 \mathrm{mV}$, 每个电压等待 1000 个脉冲, 并对单光子探测器进行累计计数. 图中圆圈是每个电压阶梯的计数
结果, 实线是正弦拟合的结果

差 $\pi$ 的电压作为 $V_{\text {ref }}$, 则由(4)式可以得到:

$$
\pi=\pi \frac{V_{c_{-} \text {min }}}{V_{\text {halfb }}}+\Delta \varphi_{0}+\Delta \varphi_{\text {var }}
$$

其中 $V_{\text {half }}$ 为调相器的半波电压, $\Delta \varphi_{\mathrm{var}}$ 即为相位漂移. 在进行密钥传输时, Alice 的 4 个调相电压工作点分别 为

$$
\begin{gathered}
\left\{V_{\mathrm{a}, 0}, V_{\mathrm{a}, 1}, V_{\mathrm{a}, 2}, V_{\mathrm{a}, 3}\right\}=\left\{V_{\mathrm{a}, 0}, V_{\mathrm{a}, \pi / 2}, V_{\mathrm{a}, \pi}, V_{\mathrm{a}, 3 \pi / 2}\right\} \\
=\left\{0, \frac{1}{2} V_{\text {half }}, V_{\text {half }}, \frac{3}{2} V_{\text {half }}\right\} .
\end{gathered}
$$

通过 $V_{\text {half }}$ 和 $V_{\text {ref }}$ 可以得到 Bob 的电压工作点.

$$
\begin{aligned}
& \left\{V_{\mathrm{b}, 0}, V_{\mathrm{b}, 1}, V_{\mathrm{b}, 2}, V_{\mathrm{b}, 3}\right\}=\left\{V_{\mathrm{b}, 0}, V_{\mathrm{b}, \pi / 2}, V_{\mathrm{b}, \pi}, V_{\mathrm{b}, 3 \pi / 2}\right\} \\
= & \left\{V_{\text {ref }}-V_{\text {half }}, V_{\text {ref }}-\frac{1}{2} V_{\text {half }}, V_{\text {ref }}, V_{\text {ref }}+\frac{1}{2} V_{\text {half }}\right\} .
\end{aligned}
$$

将(7)式应用于实际系统时，应考虑电压溢出时 的饱和处理.

当 Alice 和 Bob 分别将其调相电压设定为 $V_{\mathrm{a}, \mathrm{j}}$ 和 $V_{\mathrm{b}, \mathrm{j}}$ 时, 其相位差为

$$
\Delta \varphi=\pi \frac{V_{\mathrm{b}, \mathrm{j}}-V_{\mathrm{a}, \mathrm{i}}}{V_{\mathrm{half}}}+\Delta \varphi_{0}+\Delta \varphi_{\mathrm{var}}=\frac{j-i}{2} \pi .
$$

这一结果表明相位漂移所带来的误差得到了补 偿.

\section{3 实验装置和实验结果}

图 3 和 4 分别为实验系统的结构图和真实系统的 照片. 系统采用经过衰减的弱相干激光作为光源. 从 $1550 \mathrm{~nm}$ 激光器发出的光脉冲, 由 $1: 99$ 光纤分束器 分光, $1 \%$ 的输出作为量子信号光进入 Alice 的干涉环 并通过电控衰减器进行衰减, $99 \%$ 的输出作为同步信 号光. 量子信号经过 $37.2 \mathrm{~km}$ 的光纤信道进入接收端 的干涉环. 数据采集系统负责采集单光子探测器的 探测器结果并通过 USB2.0 接口送入主机. 主机计算 相位漂移的参数并控制调相器的工作电压进行主动相 位补偿. 系统使用 BB84协议, 脉冲重复频率为 $1 \mathrm{MHz}$. 量子信号光衰减为平均每脉冲 0.1 个光子. 信道衰减 为 $7.4 \mathrm{~dB}$. 系统使用的探测器为 idQuantique 公司生产 的 InGaAs 单光子探测器, 其暗计数为 $1.5 \times 10^{-5}$.

通过连续监测 $\mathrm{PM}_{\mathrm{a}}$ 和 $\mathrm{PM}_{\mathrm{b}}$ 保持固定电压时 $\mathrm{APD}$ 计 数的变化, 可以评估系统的相位漂移. 从图 5 可以看 出, 相位漂移没有特定的规律并且漂移的速率波动 很大, 实验中相位漂移的速度最快可以超过 4.2 $\mathrm{rad} / \mathrm{min}$, 高于Townsend等人 ${ }^{[15]}$ 和Makarov等人 ${ }^{[18]}$ 在 光学平台实验环境下给出的相位漂移数据 0.6 $\mathrm{rad} / \mathrm{min}$ 和 $2.0 \mathrm{rad} / \mathrm{min}$.

图 6 给出了系统在无人职守条件下连续运行 $18 \mathrm{~h}$ 


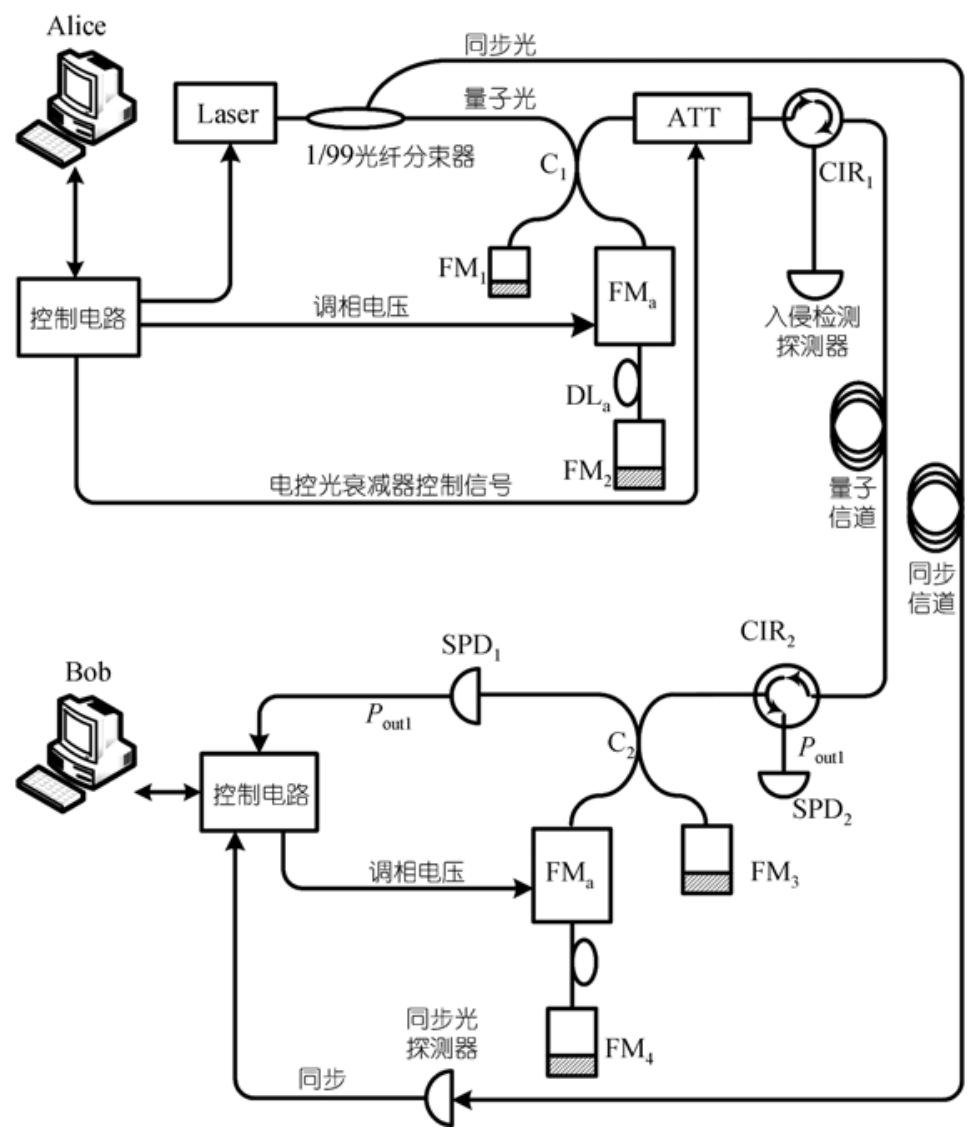

图 3 Faraday-Michelson 量子密钥传输系统实验框图

Laser 为 $1550 \mathrm{~nm}$ 激光器; FM 为法拉第镜; PM 为光学调相器; $\mathrm{C}$ 为光纤耦合器; DL 为光纤延时线; ATT 为电控光衰减器; SPD 为单光子探测器

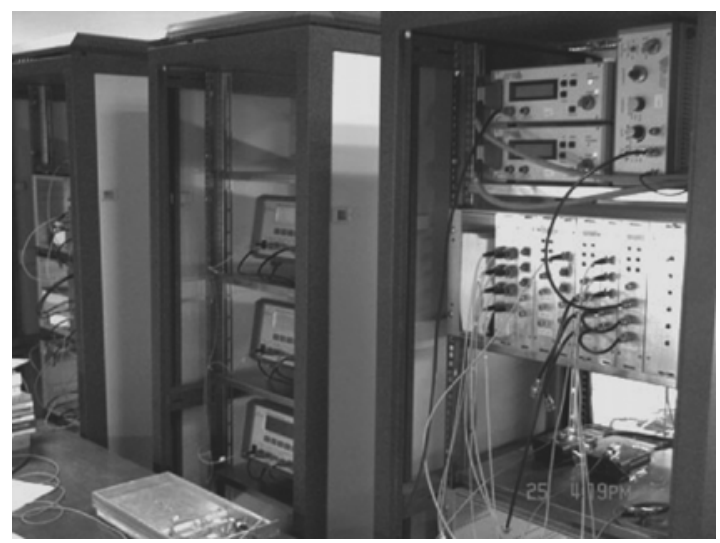

图 4 实际的量子密码系统

干涉环封装在 $5 \mathrm{U}$ 插件之中, 无特殊控温系统

所得到的误码率. 每个数据点由 $1 \mathrm{M}$ 比特的密钥数据 统计得出. 实验中使用单探头方案, 原始密钥率为 800 bps. 由相位不匹配所引起的系统误码率是用于 评估相位补偿方案的重要性能指标. 实验得到的长 期平均误码率为 $1.59 \%$, 误码的标准差为 $0.46 \%$. 系

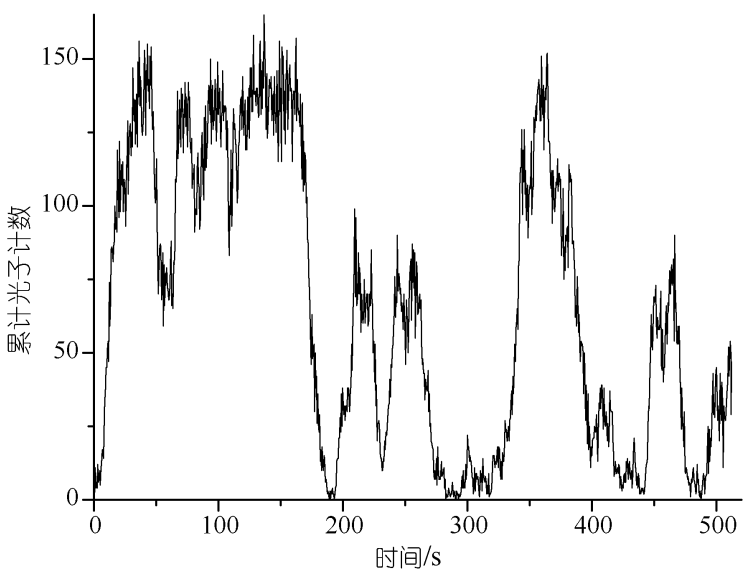

图 5 系统相位漂移

统的条纹可见度为 $98.98 \%$, 因此由系统干涉不完全 所引起的误码为 $0.51 \%$. 探测器暗计数所引起的误码 为 $0.28 \%$, 因此由相位不匹配和其他因素所引起的误 码为 $0.8 \%$. 由公式(2)可以得出，此时发射和接收端 的相位误差被控制在了 $0.2 \mathrm{rad}$ 以内. 对比图 5 和图 6 


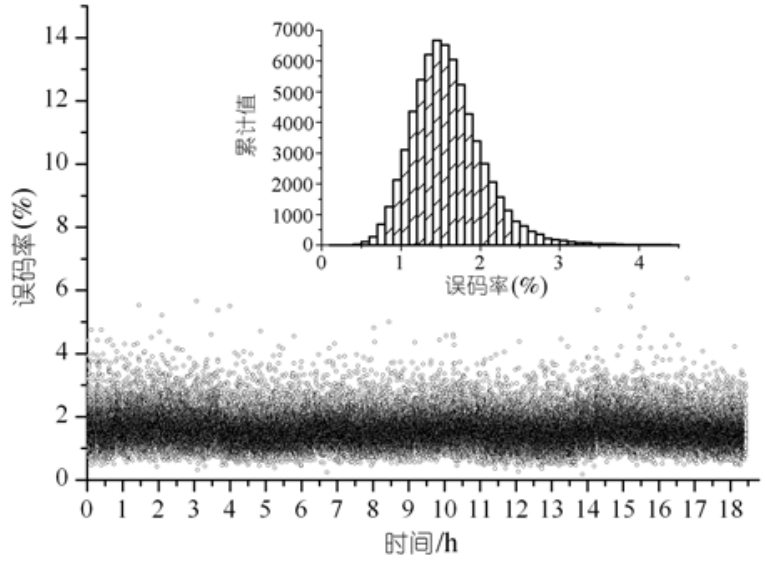

图 6 系统连续运行的误码率波动曲线 插图是误码率的统计分布

可以看出, 在系统出现大幅度的相位漂移时, 误码并 未随之出现大幅波动, 这表明主动相位补偿方案有 效的补偿了 QKD 系统实际运行时的相位漂移. 图 6 中有一些记录点的误码超过了 $4 \%$, 这是由于除温度 造成的相漂之外, 突发的强震动等因素也会造成系 统相位发生短时间的大幅度波动，超出补偿算法的 有效范围从而造成突发的高误码.

系统占空比是评估 QKD 系统效率的重要指标之 一. 这里定义系统占空比 $\tau$ 为有效密码传输时间占总 传输时间的百分比即 $\tau=t_{\text {tran }} / t_{\text {total }}$. 我们的实验中系统 占空比 $\tau$ 约为 $96.4 \%$, 并且在整个传输过程中保持恒 定.

\section{4 结论}

本文分析了实际环境下造成 Faraday-Michelson 量子密钥分配系统相位漂移的主要原因, 在此基础 上提出了一种高效的主动相位扫描和补偿方法. 在 不增加器件和温控设备的条件下, 应用这一方案系 统进行了大于 $18 \mathrm{~h}$ 的稳定运行, 平均误码率 $1.59 \%$, 系统占空比 $96.4 \%$. 实验结果表明: 应用了主动补偿 方案的 QKD 系统可以在脱离光学平台的实际工作条 件下长时间稳定运行.

\section{参考文献}

1 Bennett C H, Brassard G. Quantum cryptography: Public key distribution and coin tossing. In: Proceedings of International Conference on Computers, Systems and Signal Processing, 1984, 175 $-179$

2 Bennett C H, Bessette F, Brassard G, et al. Experiment quantum cryptography. J Crypto, 1992, 5(3): 3-28

3 Hughes R, Morgan G, Peterson C. Practical quantum key distribu- tion over a $48 \mathrm{~km}$ optical fiber network. J Mod Opt, 2000, 47: 533 $-547$

4 Stucki D, Gisin N, Guinnard O, et al. Quantum key distribution over $67 \mathrm{~km}$ with a plug \& play system. New J Phys, 2002, 4: 41.1 $-41.8[\mathrm{DOI}]$

5 Huges R, Nordholt J, Derkacs D, et al. Practical free-space quantum key distribution over $10 \mathrm{~km}$ in daylight and night. New J Phys, 2002, 4: 43.1-43.14

6 Gobby C, Yuan Z L, Shields A J. Quantum key distribution over $122 \mathrm{~km}$ of standard telecom fiber. Appl Phys Lett, 2004, 84: 3762 -3764[DOI]

7 Mo X F, Zhu B, Han Z F, et al. Faraday-Michelson system for quantum cryptography. Opt Let, 2005, 30(19): 2632-2634[DOI]

8 Gisin N, Ribordy G, Tittel W, et al. Quantum cryptography. Rev Mod Phys, 2002, 74: 145-195[DOI]

9 Han Z F, Mo X F, Gui Y Z, et al. Stability of phase-modulated quantum key distribution systems. Appl Phys Lett, 2005, 86: 221103, 1-3[DOI]

10 Zbinden H, Gautier J D, Gisn N, et al. Interferometry with Faraday mirrors for quantum cryptography. Electron Lett, 1997, 33: 586588 [DOI]

11 Muller A, Herzog T, Huttner B, et al. "Plug and play" systems for quantum cryptography. Appl Phys Lett, 1997, 70: 793-795[DOI]

12 Vakhitov A, Mkarov V, Hjelme D R. Large pulse attack as a method of conventional optical eavesdropping in quantum cryptography. J Mod Opt, 2001, 48: 2023-2038

13 Ribordy G, Brendel J, Gautier J D, et al. Long-distance entanglement-based quantum key distribution. Phys Rev A, 2001, 63: 012309[DOI]

14 Nambu Y, Hatanaka T, Nakamura K. BB84 quantum key distribution system based on silica-based planar lightwave Circuits. Jpn J Appl Phys, 2004, 43: L1109-L1110[DOI]

15 Hongjo T, Inoue K, Takahashi H. Differential-phase-shift quantum key distribution experiment with a planar light-wave circuit Mach-Zehnder interferometer. Opt Lett, 2004, 29: 2797 2799[DOI]

16 Nambu Y, Yoshino K, Tomita A. One-way quantum key distribution system based on planar lightwave circuits. Jpn J Appl Phys, 2006, 45: 5344-5348 [DOI]

17 Townsend P D, Rarity J G, Tapster P R. Single photon interferometer in $10 \mathrm{~km}$ long optical fibre interferometer. Elect Lett, 1993, 29: 634-635[DOI]

18 Yuan Z L, Shields A J. Continuous operation of a one-way quantum key distribution system over installed telecom fibre. Opt Exp, 2005, 13(2): 660-665[DOI]

19 Zavriyev A, Leverrier A, Denchev V, et al. Improving the performance of quantum key distribution apparatus. J Mod Opt, 2007, 54: 305-313[DOI]

20 Makarov V, Brylevski A, Hjelme D R. Real-time phase tracking in single-photon interferometer. Appl Opt, 2004, 43(22): 43854392[DOI]

21 Mo X F. Experimental Research on Quantum Cryptography, Doctor Dissertation. Hefei, Anhui: University of Science and Technology of China, 2006 52-19-2221 